\section{P009 THE NEED TO EDUCATE UNIVERSITY STUDENTS ABOUT SLEEP}

${ }^{1}$ Connor Qiu* ${ }^{2}$ Yizhou Yu, ${ }^{2}$ Abdullah Cheema, ${ }^{3}$ Christopher-James Harvey, ${ }^{2}$ Mary Morrell. ${ }^{1}$ St Mary's Hospital, Isle of Wight NHS Trust, Newport; 'Imperial College London, London; ${ }^{3}$ University of Oxford, Oxford

\subsection{6/bmjresp-2019-bssconf.9}

Introduction Poor sleep hygiene negatively impacts cognitive and physical abilities in students and is common among students of higher education. ${ }^{1}$ Moreover, a wide-range of literature explores the detrimental effect of poor sleep quality on learning.

Methods An anonymous, self-administered questionnaire was made available to a cohort of medical students at Imperial College London ( $n=113 ; 60$ female). Demographic information was collected to determine existing sleep quality. Questions regarding understanding of, and desire for sleep hygiene interventions to improve their experience of medical education were created on a 5-point Likert scale, ranging from 5 (strongly agree) to 1 (strongly disagree). Students were also asked to rank, the aspects in their lifestyle that warranted the most attention for improvement.

Results Students from across all years strongly agreed that their sleeping habits could be improved (4.13 \pm 0.86$)$. Equipping students with the time and energy management tools needed to maintain consistent sleep of adequate duration would be well received $(3.73 \pm 0.97)$. Students agree that a concerted intervention effort, such as having sleep promotion activities across campus would be beneficial for their education $(3.65 \pm 0.90)$.

This study identified the main factors influencing sleep quality as the latency to fall asleep, sleep duration and frequency of dreams, together accounting for $29.0 \%\left(\mathrm{r}^{2}\right)$ of the variation in sleep. The presence of a bed partner, pain, temperature, breathing problems and waking up at night did not significantly influence sleep quality.

Discussion University students would benefit from a comprehensive sleep education drive. Efforts in improving sleep quality could be directed to decreasing the latency to sleep onset and to increasing sleep duration via naps.

\section{REFERENCE}

1. Mah C D, Kezirian E J, Marcello B M, Dement W C. Poor sleep quality and insufficient sleep of a collegiate student athlete population. Sleep Health 2018;4 (3):251-257

\section{P010 DOES TONGUE REDUCTION SURGERY AFFECT SLEEP DISORDERED BREATHING IN PATIENTS WITH BECKWITH-WIEDEMANN SYNDROME?}

Ansel Godinho*, Martin Samuels, Juling Ong, Kylie Russo, Francois Abel, Aidan Laverty. Great Ormond Street Hospital for Children NHS Foundation Trust, London, UK

\subsection{6/bmiresp-2019-bssconf.10}

Introduction Beckwith-Wiedemann Syndrome (BWS) is a congenital condition where patients have macroglossia, often requiring tongue reduction surgery as well as being a potential risk factor for obstructive sleep apnoea (OSA). Prevalence of OSA is high and therefore sleep studies (SS) pre- and post-surgery are useful to assess the impact of surgery.

Method From 58 patients with BWS who underwent SS before or after tongue reduction surgery over a 20-year period, there were 9 patients who had tongue reduction surgery plus a SS pre- and post-operatively with sufficient data to calculate an Apnoea Hypopnoea Index (AHI).

Results Figure 1 shows the change in Obstructive AHI (OAHI) before and after tongue reduction surgery in the 9 patients.

Discussion In our cohort with pre- and post-surgery SS data the prevalence of OSA was 44\% (mild 100\%). 4 patients showed reductions in OAHI after surgery (mean change: 1evs/ $\mathrm{hr}$ ), 3 others showed a worsening (mean change: $5.5 \mathrm{evs} / \mathrm{hr}$ ), while 2 showed no change in OAHI. Of the 3 that worsened, 1 was restudied after two years and showed no evidence of OSA with no medical intervention. Another had grade 2 tonsils which have been surgically removed. The third patient is waiting for ENT surgical review. The small sample size of BWS patients with sufficient SS to qualify for this study may be related to the referral pathway with the majority of patients diagnosed with moderate to severe OSA pre- surgery were either treated (e.g. tracheostomy) or surgery delayed due to OSA.

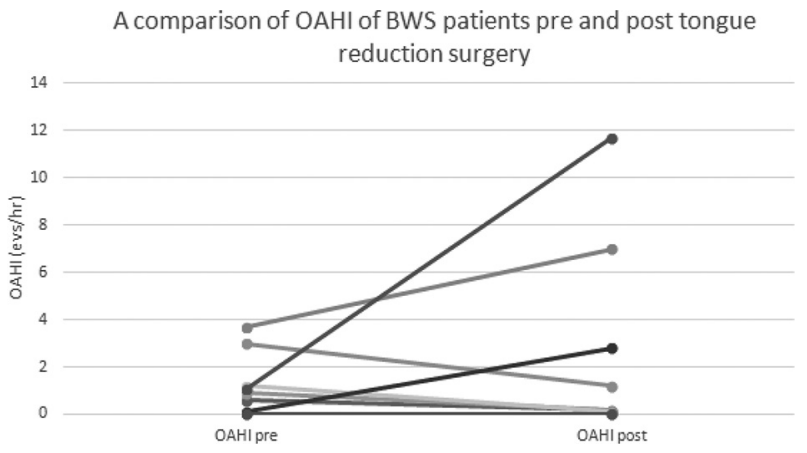

Abstract P010 Figure 1 OAHI data pre-and post-operatively

In conclusion, tongue reduction surgery does not appear to affect the level of OSA and patients who had worsening of OSA, had other factors in their history predominantly ENT related. An alternative method of assessment, such as a questionnaire, focussed on sleep disordered breathing, might be a better determinant of the impact of surgery.

\section{P011 DEVELOPMENT OF AN ONLINE BEHAVIOURAL SLEEP INTERVENTION FOR PARENTS OF CHILDREN WITH EPILEPSY, FOR USE IN THE CASTLE (CHANGING AGENDAS ON SLEEP, TREATMENT AND LEARNING IN EPILEPSY) STUDY CLINICAL TRIAL}

${ }^{1}$ Luci Wiggs*, ${ }^{1}$ Georgia Cook, ${ }^{2,3}$ Harriet Hiscock, ${ }^{4}$ Paul Gringras. ${ }^{1}$ Psychology Dept., Oxford Brookes University, Oxford, UK; ${ }^{2}$ Centre for Community Child Health, Murdoch Children's Research Institute; Health Services Research Unit, The Royal Children's Hospital, Melbourne, Australia; ${ }^{3}$ Department of Paediatrics, The University of Melbourne, Melbourne, Australia; ${ }^{4}$ Evelina London Children's Hospital, Guys and St Thomas's NHS Trust, UK

10.1136/bmjresp-2019-bssconf.11

Introduction Many of the same sleep problems experienced by children with epilepsy (CWE) are commonly seen in typically developing (TD) children. Behavioural sleep interventions (BSI) are commonly and successfully used to treat these sleep problems in TD children and in some neurodevelopmental disorder populations. BSI should therefore be effective in CWE. However, there are special seizure-related considerations for CWE and their parents which may need to be acknowledged in any BSI for this population. Through Patient and Public 